\title{
Performance Analysis of Contourlet-Based HYPERSPECTRAL IMAGE FUSION METHODS
}

\author{
Yoonsuk Choi*, Ershad Sharifahmadian, Shahram Latifi \\ Dept. of Electrical and Computer Engineering, University of Nevada, Las Vegas \\ 4505 Maryland Parkway, Las Vegas, NV 89154-4026
}

\begin{abstract}
Recently, contourlet transform has been widely used in hyperspectral image fusion due to its advantages, such as high directionality and anisotropy; and studies show that the contourlet-based fusion methods perform better than the existing conventional methods including wavelet-based fusion methods. Few studies have been done to comparatively analyze the performance of contourlet-based fusion methods; furthermore, no research has been done to analyze the contourlet-based fusion methods by focusing on their unique transform mechanisms. In addition, no research has focused on the original contourlet transform and its upgraded versions. In this paper, we investigate three different kinds of contourlet transform: i) original contourlet transform, ii) nonsubsampled contourlet transform, iii) contourlet transform with sharp frequency localization. The latter two transforms were developed to overcome the major drawbacks of the original contourlet transform; so it is necessary and beneficial to see how they perform in the context of hyperspectral image fusion. The results of our comparative analysis show that the latter two transforms perform better than the original contourlet transform in terms of increasing spatial resolution and preserving spectral information.
\end{abstract}

\section{KEYWORDS}

Contourlet, Fusion, Hyperspectral, Panchromatic

\section{INTRODUCTION}

Hyperspectral imaging is a new technique in remote sensing that collects hundreds of continuous and highly correlated spectral bands for the same area on the surface of the earth [1]. Due to the high spectral resolution of hyperspectral imaging, the ability to detect and identify individual materials or land-cover classes is greatly enhanced with regards to other traditional remote sensing techniques. However, it also brings significant challenges and difficulties in hyperspectral images processing with such richness and redundancy information. Therefore, various methods such as feature extraction and image fusion are firstly applied to reduce data dimensionality and eliminate redundancy of hyperspectral data [2]-[6]. The main purpose of these methods is to enhance the hyperspectral image analysis.

Various image fusion methods have been proposed for combining high resolution visual images with low resolution spectral images. A detailed review on this issue was given by Pohl and Van Genderen[7]. Some methods, such as intensity-hue-saturation (IHS) [8]-[9], Brovey transform (BT) [10]-[11], and principal component analysis (PCA) [11]-[12], provide superior visual highresolution multispectral images but ignore the requirement of high-quality synthesis of spectral information. While these methods are useful for visual interpretation, high-quality synthesis of spectral information is very important for most remote sensing applications based on spectral signatures, such as lithology and soil and vegetation analysis [13]. Garguet-Duport et al. [14] has shown that the high-quality synthesis of spectral information is particularly well suited in the case 
of vegetation analysis. Wald et al. [15] suggests that the fused hyperspectral images should be as identical as possible to the real hyperspectral images, the corresponding hyperspectral sensors would observe at the high-resolution level. A large amount of research has been done in terms of this constraint. The high-pass filtering (HPF) [16]-[17] and high-pass modulation (HPM) [18] methods and those of [19] and [20] have shown better performance in terms of the high-quality synthesis of spectral information. More recently, an underlying multi-resolution analysis employing the discrete wavelet transform has been used in image fusion. It was found that multisensor image fusion is a trade-off between the spectral information from a low resolution spectral image sensor and the spatial information from a high resolution visual image sensor. With the wavelet transform fusion method, it is easy to control this trade-off.

Wavelet based image fusion techniques are implemented by replacing the detail components (high frequency coefficients) from a colored input image with the detail components from another gray-scale input image. Currently used wavelet-based image fusion methods are mostly based on two computation algorithms: the Mallat algorithm [21]-[22] and the à trous algorithm [23]-[24]. The Mallat algorithm-based dyadic wavelet transform (WT), which uses decimation, is not shiftinvariant and exhibits artifacts due to aliasing in the fused image. In contrast, the à trous algorithm-based dyadic wavelet transform (ATW) method, which does not use decimation, is shift-invariant, a characteristic that makes it particularly suitable for image fusion. However, the Wavelet based fusion techniques are not optimal in capturing two-dimensional singularities from the input images. The two-dimensional wavelets, which are obtained by a tensor-product of onedimensional wavelets, are good in detecting the discontinuities at edge points. However, the 2-D Wavelets exhibit limited capabilities in detecting the smoothness along the contours [25]. Moreover, the singularity in some objects is due to the discontinuity points located at the edges. These points are located along smooth curves rendering smooth boundaries of objects. Do and Vetterli introduced the new two-dimensional Contourlet transform (CT) [26]. This transform is more suitable for constructing a multi-resolution and multi-directional expansions using nonseparable Pyramid Directional Filter Banks (PDFB) with small redundancy factor [27].

Do group has developed two more contourlet transforms based on the original contourlet transform theory. The first one is called nonsubsampled contourlet transform (NSCT) and the second one is called contourlet transform with sharp frequency localization (CTSFL). Both of them were developed to overcome the major drawbacks of the original contourlet transform. Researchers have recently proposed new contourlet transform based fusion methods that outperform the other existing methods. When a new fusion method is proposed, it is necessary to go through comparative analyses in order to validate the newly proposed method. Moreover, performance analysis studies take an important role in the field of image fusion because it gives a right direction in developing a better fusion method. However, no research has been conducted in analyzing the performance of three contourlet transforms. In this paper, we mainly focus on the performance analysis of the three contourlet transforms using the same fusion rule but different multi-scale decompositions. In Section 2, three different contourlet transforms are discussed in detail to provide a clear overview on their background theories, advantages and drawbacks. Next, the fusion framework and fusion scheme used in our experiments are explained in Section 3. Experimental study and analysis are discussed in Section 4, and we conclude in Section 5.

\section{Contourlet Transform}

\subsection{Original Contourlet Transform}

The wavelet transform is good at isolating the discontinuities at object edges, but cannot detect the smoothness along the edges. Moreover, it can capture limited directional information. The contourlet transform can effectively overcome the disadvantages of wavelet; contourlet transform 
is a multi-scale and multi-direction framework of discrete image. In this transform, the multiscale analysis and the multi-direction analysis are separated in a serial way. The Laplacian pyramid (LP) [28] is first used to capture the point discontinuities, then followed by a directional filter bank (DFB) [29] to link point discontinuities into linear structures. The overall result is an image expansion using basic elements like contour segments. The framework of contourlet transform is shown in Figure 1.

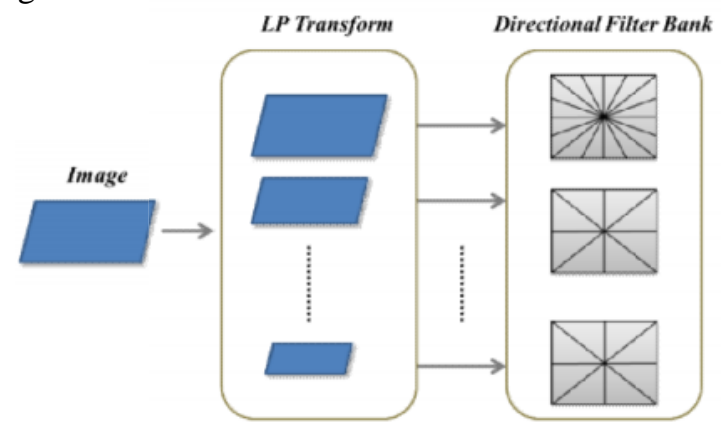

Figure 1. The contourlet transform framework.

Figure 2 shows the contourlet filter bank. First, multi-scale decomposition is performed by the Laplacian pyramid, and then a directional filter bank is applied to each band pass channel.

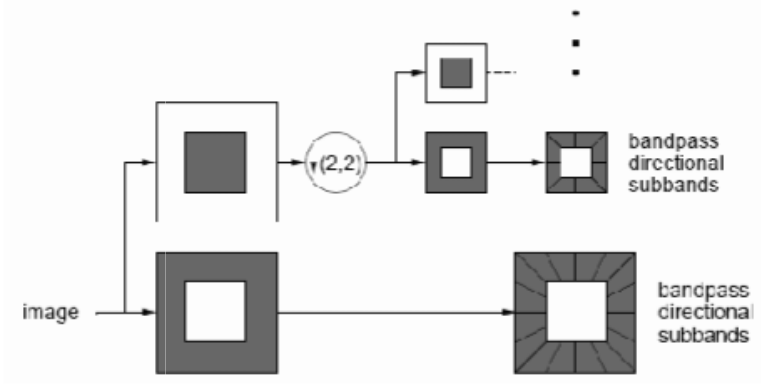

Figure 2.The contourlet filter bank.

Contourlet expansion of images consists of basis images oriented at various directions in multiple scales with flexible aspect ratio. In addition to retaining the multi-scale and time-frequency localization properties of wavelets, the contourlet transform offers high degree of directionality. Contourlet transform adopts nonseparable basis functions, which makes it capable of capturing the geometrical smoothness of the contour along any possible direction. Compared with traditional image expansions, contourlet can capture 2-D geometrical structure in natural images much more efficiently [30].

Furthermore, for image enhancement, one needs to improve the visual quality of an image with minimal image distortion. Wavelet-based methods present some limitations because they are not well adapted to the detection of highly anisotropic elements such as alignments in an image. Contourlet transform (CT) has better performance in representing the image salient features such as edges, lines, curves and contours than wavelet transform because of CT's anisotropy and directionality. Therefore, CT is well-suited for multi-scale edge based image enhancement.

To highlight the difference between the wavelet and contourlet transform, Figure 3 shows a few wavelet and contourlet basis images. It is possible to see that contourlets offer a much richer set of directions and shapes, and thus they are more effective in capturing smooth contours and geometric structures in images. 
International Journal on Information Theory (IJIT), Vol.2, No.1/2/3/4, October 2013
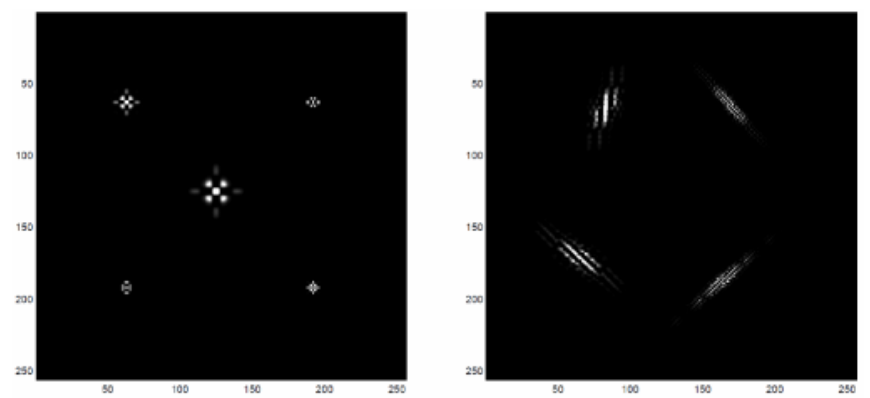

Figure 3. Comparison between actual 2-D wavelets (left) and contourlets (right) [3].

\subsection{Nonsubsampled Contourlet Transform}

The contourlet transform proposed by Do and Vetterli is a real two-dimensional transform, which is based on nonseparable filter banks and provides an efficient directional multi-resolution image representation. The contourlet transform (CT) expresses image by first applying a multi-scale transform (the Laplacian pyramid transform), followed by a direction filter banks (DFB) to link point discontinuities into linear structures. The contourlets satisfy anisotropy principle and can capture intrinsic geometric structure information of images and achieve better expression than discrete wavelet transform, especially for edges and contours. However, because of the downsampling and upsampling, the CT lacks of shift-invariance and results in ringing artifacts. The shift-invariance is desirable in image analysis applications, such as edge detection, contour characterization, image fusion, etc. So Cunha et al. proposed nonsubsampled contourlet transform [31] based on nonsubsampled pyramid decomposition and nonsubsampled filter bank (NSFB). In NSCT, the multi-scale analysis and the multi-direction analysis are also separate, but both are shift-invariant. First, the nonsubsampled pyramid (NSP) is used to obtain a multi-scale decomposition by using two-channel nonsubsampled 2-D filter bands. Second, the nonsubsampled directional filter bank is used to split band pass sub-bands in each scale into different directions. Figure 4 shows two-level decomposition by using a combination of a NSP and NDFB. Because of no downsampling in pyramid decomposition, the lowpasssubband has no frequency aliasing, even the bandwidth of lowpass filter is larger than $\pi / 2$; hence, the NSCT has better frequency characteristics than CT.

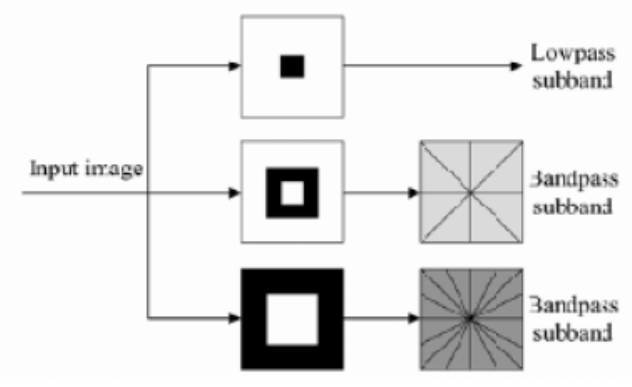

(a)

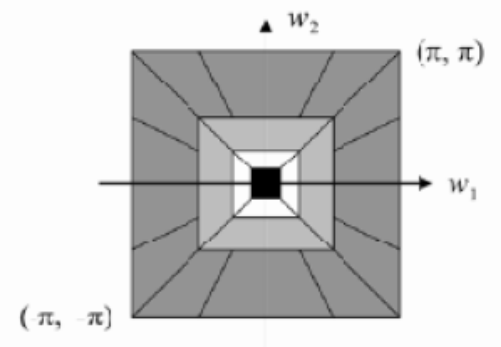

(b)

Figure 4. Two level nonsubsampled contourlet transform decomposition [32]. (a) NSFB structure that implements NSCT. (b) Corresponding frequency partition. 


\subsection{Contourlet Transform with Sharp Frequency Localization}

As discussed earlier, the contourlet transform was proposed as a directional multi-resolution image representation; however, the major drawback of the original contourlet construction is that its basis images are not localized in the frequency domain.

The contourlet transform is constructed as a combination of the Laplacian pyramid and the directional filter banks (DFB). Conceptually, the flow of operation can be illustrated by Figure 5(a), where the Laplacian pyramid iteratively decomposes a 2-D image into lowpass and highpasssubbands, and the DFB are applied to the highpass sub-bands to further decompose the frequency spectrum. Using ideal filters, the contourlet transform will decompose the 2-D frequency spectrum into trapezoid-shaped regions as shown in Figure 5(b).

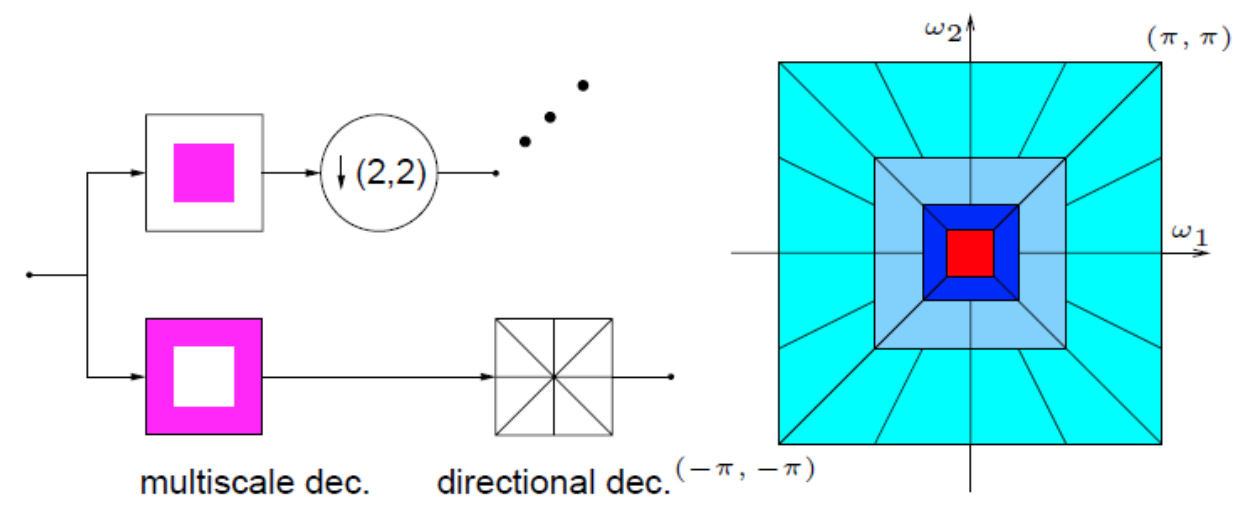

(a)

(b)

Figure 5. The original contourlet transform. (a) Block diagram. (b) Resulting frequency division.

In practice, when non-ideal filters are used, the resulting contourlets do not have the desired sharp frequency domain localization. Although the majority of the energy in each subband is still concentrated on the ideal support regions, there are also significant amount of aliasing components showing up at locations far away from the desired support. This kind of frequency aliasing is undesirable, since the resulting contourlets in the spatial domain are not smooth along their main ridges and exhibit some fuzzy artifacts. Consequently, this jeopardizes the efficiency of contourlets in representing smooth boundaries in natural images [33]. This phenomenon has also been independently observed by other researchers, e.g., in the work by Candes et al. [34].

Y. Lu and M. N. Do proposed a new method of constructing the contourlet transform to overcome the drawback [33]. Figure 6 is a new construction of the contourlet transform. Directional filter bank is still used for directional decomposition. However, an important distinction from the original contourlet transform is that, instead of using the Laplacian pyramid, a new pyramid structure for the multi-scale decomposition is used and it is conceptually similar to the one used in the steerable pyramid [35]. As an important difference from the Laplacian pyramid, the new multi-scale pyramid can employ a different set of lowpass and highpass filters for the first level and all other levels [33]. 


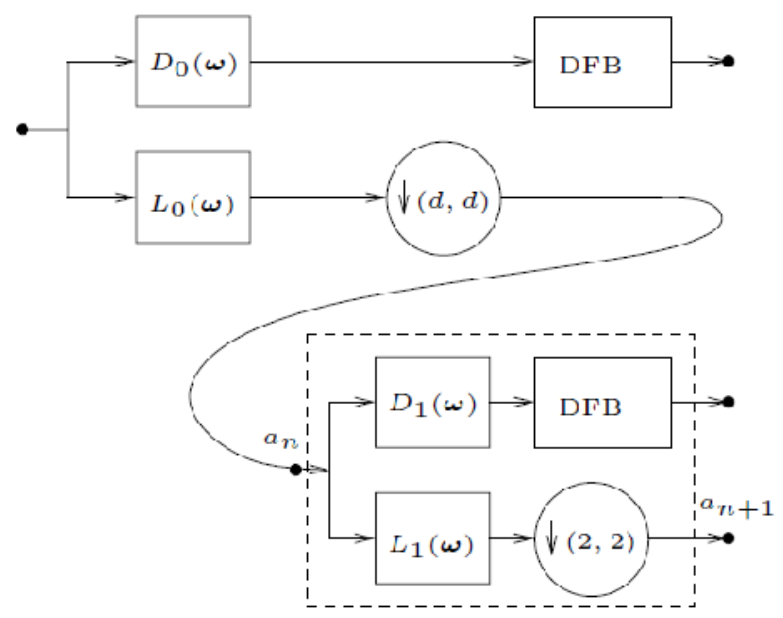

Figure 6 .The new block diagram. Only the analysis part is shown, while the synthesis part is exactly symmetric [33].

\section{HYPERSPECTRAL IMAGE FUSION}

Hyperspectral image analysis is used in various applications due to its advantages; however, the main drawback is its low spatial resolution. Many ideas have been developed to enhance the hyperspectral image analysis, but the most well-known technique is the fusion of hyperspectral image with panchromatic image, also called pan-sharpening. This method increases the spatial resolution of hyperspectral image. In the process of image fusion, various theories are applied, such as Intensity-Hue-Saturation, Principle Component Analysis, Wavelet transform, Curvelet transform, and so on. Recent studies show that contourlet transform based fusion outperforms the existing methods. Therefore, the key point in our comparative analysis is contourlet transform, and we want to compare it to its successors (NSCT and CTSFL) to see how they perform in image fusion. In this section, we provide fusion framework and scheme that are used in our comparative analysis to test the original CT and its successors.

\subsection{Analysis of the Contourlet Transform Coefficients}

The distribution of the coefficients of contourlet transformis not well-regulated as good as that of wavelet transform. It isrelated to the parameter nlevels given in the DFB stagedecomposition. nlevelsis a one dimensional vector. It is used tostore the parameters of the decomposition level of each level ofpyramid for DFB. If the parameter of the decomposition level is0 for DFB, DFB will use the wavelet to process the subimageof pyramid. If the parameter is $l j$. The decomposition levels ofDFB is $2^{l j}$, which means that the subimage is divided into $2^{l j}$ directions.Corresponding to the vector parameter nlevels, thecoefficients, $Y$, of the contourlet decomposition is a vector, too.The length of $Y$ is equal to length (nlevels) +1 . $Y\{1\}$ is thesubimage of the low frequency. $\boldsymbol{Y}\{\mathbf{i}\}(\boldsymbol{i}=\mathbf{2}, \ldots$, Len $)$ is thedirection subimage obtained by DFB decomposition, i denotes the $i$ thlevel pyramid decomposition. The subband images of contourlet decomposition coefficients for famous Peppers image areshown in Figure 7[36]. 


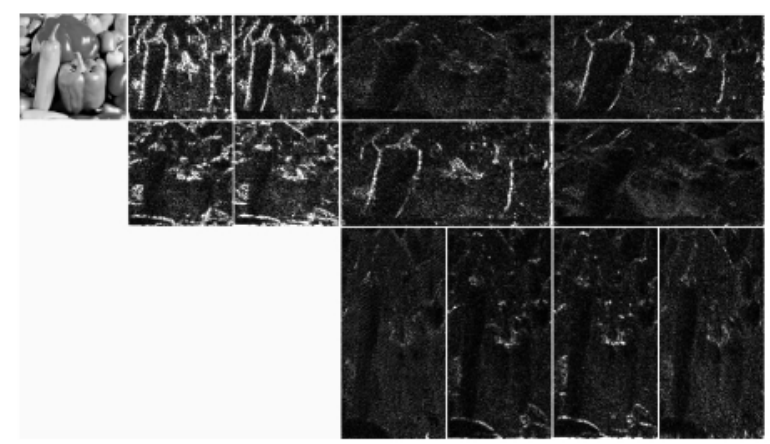

Figure 7. Example of the contourlet transform on Peppers image. Each image is decomposed into two pyramidal levels, which are then decomposed into 4 and 8 directional subbands. Small coefficients are shown in black while large coefficients are shown in white.

\subsection{Fusion Framework}

The fusion framework used in this paper is shown below in Figure 8. However, three different contourlet transforms are implemented within the framework: i) original contourlet transform (CT), ii) nonsubsampled contourlet transform (NSCT) and iii) contourlet transform with sharp frequency localization (CTSFL).

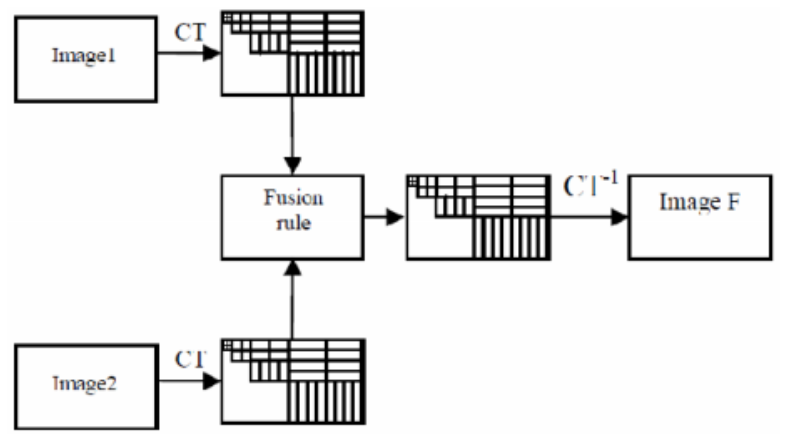

Figure 8. Fusion framework based on contourlet transforms.

\subsection{Fusion Scheme}

The fusion scheme implemented in our performance analysis is explained below. Note that the overall fusion scheme is identical except for the decomposition step because three different contourlet transforms are used.

1) The first step is to geometrically register two source images, in our case, hyperspectral image and panchromatic image. However, it takes more computational load to process the image registration. That is why many papers assume that the source images are coregistered. Also, it is not easy to get a pair of hyperspectral and panchromatic images of the same scene taken at the same time with the same viewpoint. Therefore, preprocessing is performed as follows:

The original hyperspectral image is shifted, in both horizontal and vertical directions, to produce a shifted hyperspectral image, and then the sequence was convolved with Gaussian smooth filter point-spread function (PSF) of size $3 \times 3$ with variance equal to 0.5 . Then, it is downsampled in both horizontal and vertical directions by factor of two. Lastly, zero-mean Gaussian noise was 
added to the sequence. The final result is a synthesized hyperspectral image that can be used in our experiment as a source image.

In order to get another source image, panchromatic image, the original hyperspectral image is spectrally integrated over the entire spectral range. The final result is a synthesized panchromatic image that can be used as the second source image [37].

2) After pre-processing, the fusion rule follows the method proposed by M. Qiguang and W. Baoshu[36]. The two images are decomposed in multi-scale and multi-direction by each of the three contourlet transforms. The transform coefficients of image A and B are $\boldsymbol{Y}_{\boldsymbol{A}}$ and $\boldsymbol{Y}_{\boldsymbol{B}}$, respectively. Denote the transform coefficients of the fused image by $\boldsymbol{Y}_{\boldsymbol{F}}$. Then, process the coefficients based on the following rules:

a) For the coefficients of the low frequency, fusion with the average rule:

$$
Y_{F}\{1\}=\left\{Y_{A}\{1\}+Y_{B}\{1\}\right\} / 2
$$

b) For the coefficients of the high frequency, if $\boldsymbol{l}_{i}$, the decomposition level parameters of directional filter bank in the vector nlevels, is not equal to 0 , fuse by the rule of selecting the coefficients of greater region energy using region consistency check:

$$
D_{X}(i, j)=\sum_{i \leq M, j \leq N}\left(Y_{X}(i, j)\right)^{2}, X=A, B
$$

The region energy of each coefficient of every subband is calculated by Eq. (2). With Eq. (3), we can construct the high frequency subbands of the fused image. Also, at the same time, the fusiondecision map is obtained by Eq. (4).

$$
\begin{aligned}
& Y_{F}(i, j)=\left\{\begin{array}{l}
Y_{A}(i, j), D_{A}(i, j) \geq D_{B}(i, j) \\
Y_{B}(i, j), D_{A}(i, j)<D_{B}(i, j)
\end{array}\right. \\
& \operatorname{Map}(i, j)=\left\{\begin{array}{l}
1, D_{A}(i, j) \geq D_{B}(i, j) \\
0, D_{A}(i, j)<D_{B}(i, j)
\end{array}\right.
\end{aligned}
$$

Finally, the region consistency check is done based on the fusion-decision map. If a pixel is to come from the source image A but with the majority of its surrounding neighbors from $\mathrm{B}$, this pixel will be switched to come from B.

c) If $l_{i}(i=1,2, \cdots$, Len $)$ is equal to 0 , it means that the wavelet decomposition is used in the $j$-th level image. In this case, the coefficients of wavelet decomposition should be processed with different rule for low and high frequency components. For low frequency components, fuse with the average rule:

$$
Y_{F}^{L}\{i\}=\left\{Y_{A}^{L}\{i\}+Y_{B}^{L}\{i\}\right\} / 2
$$

For horizontal, vertical and diagonal high frequency components, fuse by the rule of choosing the ones with greater region energy using the region consistency check. The procedure is identical to the Eqs. (2)-(4). The contourlet coefficients of the fused image, $Y_{F}$ is obtained this way. 
International Journal on Information Theory (IJIT), Vol.2, No.1/2/3/4, October 2013

3) The contourlet inverse transform, also called reconstruction, is done using the coefficients $Y_{F}$. The reconstructed image is the final fused image. In fact, if nlevels $=[0,0, \cdots, 0]$, it means that the Laplacian Pyramid of contourlet transform is first used, and then followed by the wavelet transform to every level of the pyramid. The fused result will be a combination of the contourlet transform and the wavelet transform. On the other hand, if $l_{i}[i=1,2, \cdots$, Len $]$ is not equal to 0 , for the subimage of each level of the Laplacian Pyramid, only the directional filter bank (DFB) decomposition is processed. In this case, the wavelet transform is not used.

\section{EXPERIMENTAl STUDY AND ANALYSIS}

In our experiments, three different contourlet transforms are used in fusing the source images with the parameter levels of $[0,2,3,4]$. As described in Section 3, we synthesized the original hyperspectral image to create two source images: i) hyperspectral image and ii) panchromatic image. Performance of the final fused images is evaluated by comparing the results with the original hyperspectral image. By using synthesized images as source image, we can easily compare the fusion results with the original reference image, and the source images (hyperspectral and panchromatic images) are perfectly registered to each other without going through image registration process. Therefore, the errors we might encounter due to wrong registration of the source images can be completely avoided.

There are many different performance evaluation metrics available in literatures to analyze pansharpenedimages [38]-[41]. These quality metrics are divided into mainly three categories: i) spatial quality measures, ii) spectral quality measure and iii) average measures to analyze the effect of both simultaneously. In terms of spatialquality analysis, correlation coefficient (CC), distortion extent (DE), root mean square error (RMSE) and Universal image quality index (UIQI) are often employed[38], [41]. In order to analyze the fusion results in terms of spectral information, relative global error in synthesis (ERGAS), spectral angle mapper (SAM) andrelative average spectral error(RASE) are often used. Root cross entropy(RCE) or mean cross entropy (MCE) and signal to noise ratio(SNR) [42]can be used to analyze both quality factors. The average gradient (AG) and standard deviation (SD) parameters don't require any reference image for evaluation. All theseparameters are explained in the literatures in detail.

Figures 9(a) and 11(a) show the original hyperspectral images that are used as a reference. These hyperspectral images were obtained from MultiSpec $\odot$ by Purdue University [43]. Next, Figures 9(b)-(c) and 11(b)-(c) show two source images (panchromatic and hyperspectral images) that have been synthesized from the original hyperspectral image as described in Section 3. Figures 10 and 12 show the fusion results based on three different contourlet transforms: i) CT, ii) NSCT and iii) CTSFL. Fusion results are compared using various quality assessment parameters. We employed correlation coefficient (CC), relative average spectral error (RASE) and spectral angle mapper (SAM) for spectral analysis. To spatially analyze the fusion results, we employed distortion extent (DE), universal image quality index (UIQI) and signal to noise ratio (SNR). 
International Journal on Information Theory (IJIT), Vol.2, No.1/2/3/4, October 2013

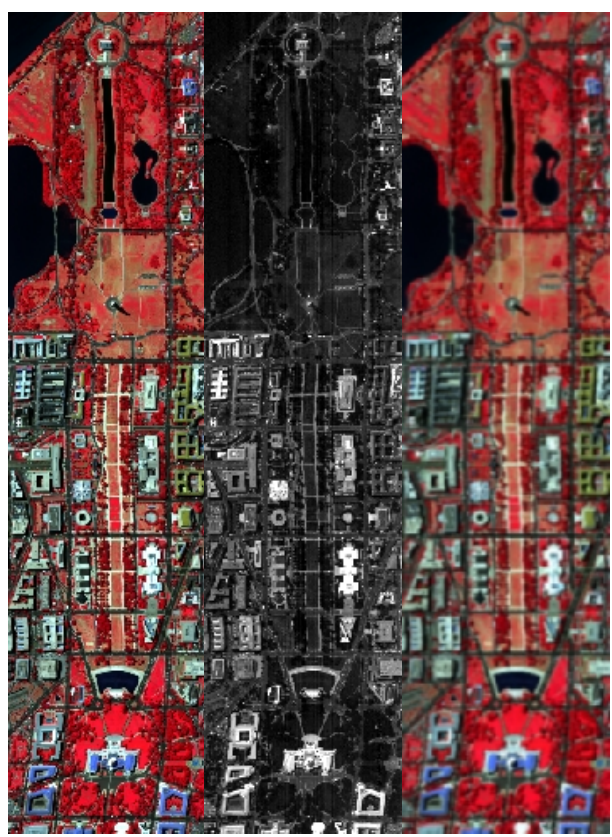

(a)

(b)

(c)

Figure 9.The original hyperspectral image and two synthesized source images. (a) Original hyperspectral image. (b) Synthesized panchromatic source image. (c) Synthesized hyperspectral image.

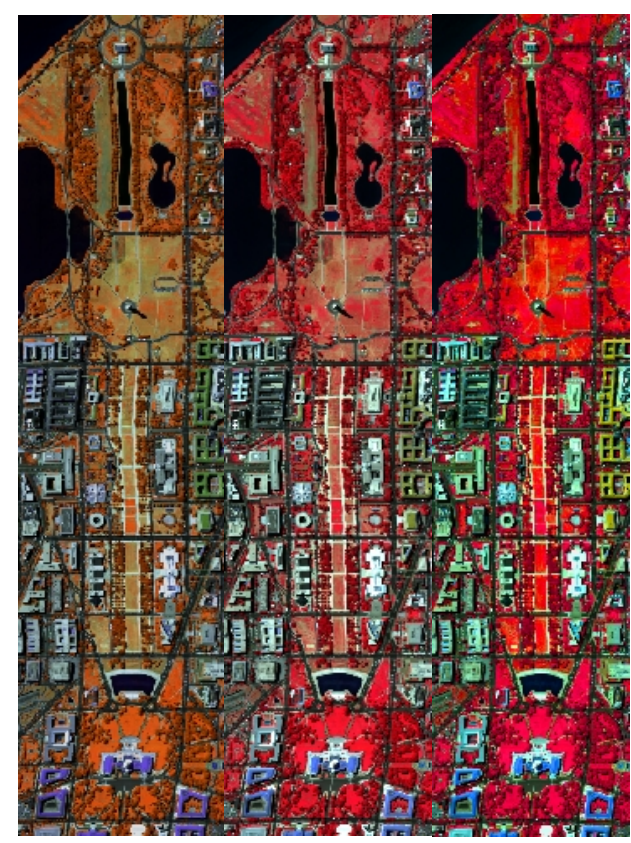

(a)

(b)

(c)

Figure 10.Fusion results. (a) Contourlet transform (CT). (b) Nonsubsampled contourlet transform (NSCT).

(c) Contourlet transform with sharp frequency localization (CTSFL). 
International Journal on Information Theory (IJIT), Vol.2, No.1/2/3/4, October 2013

Table 1. A performance comparison using quality assessment parameters.

\begin{tabular}{|c|c|c|c|c|c|c|}
\hline \multirow{2}{*}{$\begin{array}{c}\text { Transform } \\
\text { Type }\end{array}$} & \multicolumn{3}{|c|}{ Spectral Analysis } & \multicolumn{3}{c|}{ Spatial Analysis } \\
\cline { 2 - 7 } CT & CC & RASE & SAM & Distortion & UIQ & SNR \\
\hline NSCT & 0.865 & 45.764 & 0.244 & 27.207 & 0.759 & 68.872 \\
\hline CTSFL & 0.884 & 43.721 & 0.216 & $\mathbf{2 3 . 2 4 2}$ & $\mathbf{0 . 8 0 1}$ & $\mathbf{7 1 . 2 6 4}$ \\
\hline
\end{tabular}

In order to increase the reliability of our experiments, we tested with one more data set. This gives us a more clear view when analyzing the fusion results.

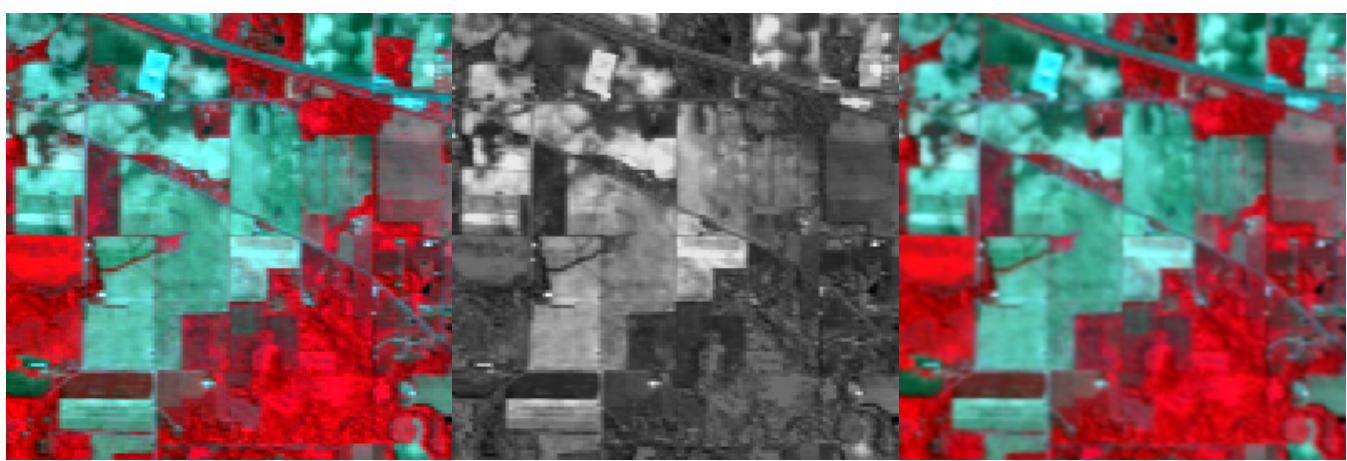

(a)

(b)

(c)

Figure 11.The original hyperspectral image and two synthesized source images. (a) Original hyperspectral image. (b) Synthesized panchromatic source image. (c) Synthesized hyperspectral image.

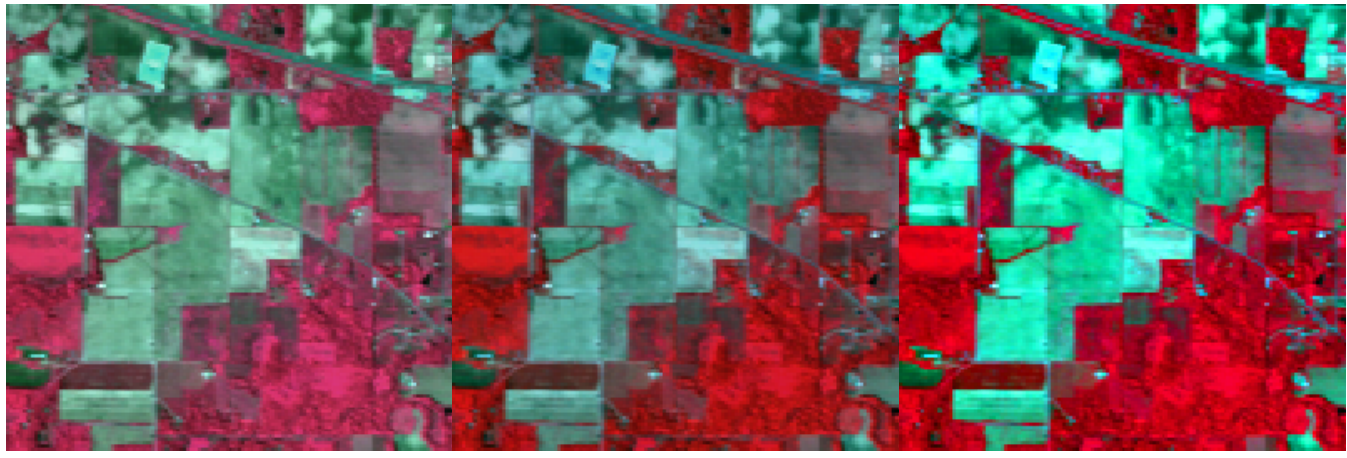

(a)

(b)

(c)

Figure 12.Fusion results. (a) Contourlet transform (CT). (b) Nonsubsampled contourlet transform (NSCT). (c) Contourlet transform with sharp frequency localization (CTSFL). 
International Journal on Information Theory (IJIT), Vol.2, No.1/2/3/4, October 2013

Table 2. A performance comparison using quality assessment parameters.

\begin{tabular}{|c|c|c|c|c|c|c|}
\hline \multirow{2}{*}{$\begin{array}{c}\text { Transform } \\
\text { Type }\end{array}$} & \multicolumn{3}{|c|}{ Spectral Analysis } & \multicolumn{3}{c|}{ Spatial Analysis } \\
\cline { 2 - 7 } CT & CC & RASE & SAM & Distortion & UIQ & SNR \\
\hline NSCT & 0.776 & 43.865 & 0.277 & 28.136 & 0.658 & 68.726 \\
\hline CTSFL & $\mathbf{0 . 7 9 8}$ & $\mathbf{4 3 . 6 5 2}$ & $\mathbf{0 . 2 4 5}$ & 26.264 & 0.662 & 68.731 \\
\hline
\end{tabular}

As we can see from Table 1 and Table 2, the fusion results show that nonsubsampled contourlet transform (NSCT) performs better in terms of increasing spatial resolution; however, contourlet transform with sharp frequency localization (CTSFL) performs better in terms of preserving spectral information. In other words, CTSFL distorts the spectral information as less as possible. Moreover, the fusion results show that NSCT and CTSFL outperform the original contourlet transform both spatially and spectrally. Contourlet transform is used in various image related applications; however, we can tell that upgraded versions of the original contourlet transform are a better choice especially in the field of image fusion. We selected hyperspectral image fusion method to test three different contourlet transforms; however, the main purpose of this research is to find a good solution to enhance the hyperspectral image analysis. Based on the results, it is obvious that the successors of the original contourlet transform are potential solutions to future development in the hyperspectral image enhancement.

\section{CONCLUSIONS}

Hyperspectral image analysis plays a very important role in many image-related applications; however, hyperspectral images have relative low spatial resolution. Therefore, it is necessary to develop new methods to enhance the hyperspectral image analysis. Among various enhancing methods, we chose pan-sharpening technique, fusing hyperspectral image with high resolution panchromatic image, for our comparative analysis because it greatly increases the spatial resolution. Next, we selected contourlet transform to use in our fusion process due to its advantages in image processing, and recent studies show that the contourlet transform outperforms other conventional hyperspectral fusion methods including wavelet transform. Therefore, our goal was to test the original contourlet transform and its two upgraded versions together, and find out which one would be a good choice in our future research on development of new hyperspectral image fusion method. The comparative analysis shows that the upgraded versions of contourlet transform (NSCT and CTSFL) outperform the original contourlet transform both spatially and spectrally. Also, NSCT shows good results spatially, and CTSFL performs better spectrally. Therefore, in our future research, we can utilize the advantages of both NSCT and CTSFL in developing a novel hyperspectral fusion method.

\section{ACKNOWLEDGEMENTS}

The Nevada EPSCoR program is funded in part by the National Science Foundation (NSF) award \#EPS-IIA-1301726 and in part by the DOD-DTRA Grant/Award \#: HDTRA1-12-1-0033. 
International Journal on Information Theory (IJIT), Vol.2, No.1/2/3/4, October 2013

\section{REFERENCES}

[1] S. A. Robila, "Using Spectral Distances for Speedup in Hyperspectral Image Processing", International Journal of Remote Sensing, Vol. 26, No. 24, 2005.

[2] N. Kambhatla and R. A. Leen, "Dimension reduction by local principal component analysis", Neural Computation, vol. 9, pp. 1493-1516, October 1997.

[3] D. Manolakis and D. Marden, "Dimensionality reduction of hyperspectral imaging data using local principal components transforms", Proceedings of SPIE, vol. 5425, pp. 393-401, April 2004.

[4] D. F. Michael and R. M. Mersereau, "On the impact of PCA dimension reduction for hyperspectral detection of difficult targets", IEEE Geoscience and Remote Sensing Letters, vol. 2, pp. 192-195, April 2005.

[5] Huang Rui and He Mingyi., "Band selection based on feature weighting for classification of hyperspectral data", IEEE Geoscience and Remote Sensing Letter, vol. 2, pp. 156-159, April 2005.

[6] JiaXiuping and J. A. Richards, "Segmented principal components transformation for efficient hyperspectral remote-sensing image display and classification", IEEE Geoscience and Remote Sensing, vol. 37, pp. 538-542, Janurary 1999.

[7] C. Pohl and J. L. Van Genderen, "Multi-sensor image fusion in remote sensing: Concepts, methods, and applications," Int. J. Remote Sens., vol. 19, no. 5, pp. 823-854, 1998.

[8] P. S. Chavez and J. A. Bowell, "Comparison of the spectral information content of Landsat thematic mapper and SPOT for three different sites in the Phoenix, Arizona region," Photogramm.Eng. Remote Sens., vol.54, no. 12, pp. 1699-1708, 1988.

[9] W. J. Carper, T. M. Lillesand, and R. W. Kiefer, "The use of Intensity-Hue-Saturation transform for merging SPOT panchromatic and multispectral image data," Photogramm.Eng. Remote Sens., vol. 56, no. 4, pp. 459-467, 1990.

[10] A. R. Gillespie, A. B. Kahle, and R. E. Walker, "Color enhancement of highly correlated images-II. Channel ratio and 'chromaticity' transformation techniques," Remote Sens. Environ., vol. 22, pp. 343-365, 1987.

[11] J. Zhou, D. L. Civco, and J. A. Silander, "A wavelet transform method to merge Landsat TM and SPOT panchromatic data," Int. J. Remote Sens., vol. 19, no. 4, pp. 743-757, 1998.

[12] P. S. Chavez and A. Y. Kwarteng, "Extracting spectral contrast in Landsat Thematic Mapper image data using selective principle component analysis," Photogramm.Eng. Remote Sens., vol. 55, no. 3, pp. 339-348, 1989.

[13] J. G. Liu, "Smoothing filter-based intensity modulation: A spectral preserve image fusion technique for improving spatial details," Int. J. Remote Sens., vol. 21, no. 18, pp. 3461-3472, 2000.

[14] B. Garguet-Duport, J. Girel, J.-M.Chassery, and G. Pautou, "The use of multi-resolution analysis and wavelets transform for merging SPOT panchromatic and multi-spectral image data," Photogramm. Eng. Remote Sens., vol. 62, no. 9, pp. 1057-1066, 1996.

[15] L. Wald, T. Ranchin, and M. Mangolini, "Fusion of satellite images of different spatial resolutions: Assessing the quality of resulting images," Photogramm. Eng. Remote Sens., vol. 63, no. 6, pp. 691699, 1997.

[16] R. A. Schowengerdt, "Reconstruction of multi-spatial, multi-spectral image data using spatial frequency content," Photogramm. Eng. Remote Sens., vol. 46, no. 10, pp. 1325-1334, 1980.

[17] P. S. Chavez, S. C. Sides, and J. A. Anderson, "Comparison of three different methods to merge multi-resolution and multi-spectral data: Landsat TM and SPOT panchromatic," Photogramm. Eng. Remote Sens., vol. 57, no. 3, pp. 295-303, 1991.

[18] R. A. Schowengerdt, Remote Sensing: Models and Methods for Image Processing, 2nd ed. Orlando, FL: Academic, 1997.

[19] J. G. Liu and J. M. Moore, "Pixel block intensity modulation: Adding spatial detail to TM band 6 thermal imagery," Int. J. Remote Sens., vol. 19, no. 13, pp. 2477-2491, 1998.

[20] Y. Zhang, "A new merging method and its spectral and spatial effects," Int. J. Remote Sens., vol. 20, no. 10, pp. 2003-2014, 1999.

[21] S. Mallat, "A theory for multi-resolution signal: The wavelet representation," IEEE Trans. Pattern Anal. Mach. Intell., vol. 11, no. 7, pp. 674-693, Jul. 1989.

[22] D. A. Yocky, "Image merging and data fusion by means of the discrete two-dimensional wavelet transform," J. Opt. Soc. Amer. A, vol. 12, no. 9, pp. 1834-1841, 1995.

[23] M. J. Shensa, "The discrete wavelet transform:Wedding the à Trousand Mallat algorithms," IEEE Trans. Signal Process., vol. 40, no. 10, pp. 2464-2482, Oct. 1992. 
[24] J. Núñez, X. Otazu, O. Fors, A. Prades, V. Palà, and R. Arbiol, "Multiresolution-based image fusion with additive wavelet decomposition," IEEE Trans. Geosci. Remote Sens., vol. 37, no. 3, pp. 12041211, May 1999.

[25] G. V. Welland, Beyond Wavelets.Academic Press, 2003.

[26] M. N. Do and M. Vetterli, "The contourlet transform: An efficient directional multiresolution image representation," IEEE Transactions on Image Processing, vol. 14, no. 12, pp. 2091-2106, 2005.

[27] S. Ibrahim and M. Wirth, "Visible and IR Data Fusion Technique Using the Contourlet Transform", International conference on computational science and engineering, CSE 09, IEEE, vol. 2, pp. 42-47, 2009.

[28] Burt P J., "Merging images through pattern decomposition", Proceedings of SPIE, 575: 173-18, 1985.

[29] Bamberger R H., "A filter bank for the directional decomposition of images: Theory and design", IEEE Trans. Signal Processing, 40 (4): 882 -893, 1992.

[30] Aboubaker M. ALEjaily et al., "Fusion of remote sensing images using contourlet transform", Innovations and Advanced Techniques in Systems, Computing Sciences and Software Engineering, Springer, pp. 213-218, 2008.

[31] Da Cunha A L, Zhou J , Do M N, "The nonsubsampled contourlet transform: theory, design , and applications", IEEE Trans . Image Proc. ,15 (10) : 308923101, 2006.

[32] Ding LI, "Remote sensing image fusion based on nonsubsampled contourlet transform and PCA", International Conference on Computer Tech and Development, 2009.

[33] Yue Lu, M.N. Do, "A New Contourlet Transform with Sharp Frequency Localization," Image Processing, 2006 IEEE International Conference on , vol., no., pp.1629,1632, 8-11 Oct. 2006.

[34] E. J. Cand es, L. Demanet, D. L. Donoho, and L. Ying, "Fast discrete curvelet transforms," Tech. Rep., Applied and Computational Mathematics, California Institute of Technology, 2005.

[35] E. P. Simoncelli,W. T. Freeman, E. H. Adelson, and D. J. Heeger, "Shiftablemultiscale transforms," IEEE Trans. Inform. Th., Special Issue on Wavelet Transforms and Multiresolution Signal Analysis, vol. 38, no. 2, pp. 587-607, March 1992.

[36] Miao Qiguang; Wang Baoshu, "A Novel Image Fusion Method Using Contourlet Transform," Communications, Circuits and Systems Proceedings, 2006 International Conference on, vol.1, no., pp.548,552, 25-28, June 2006.

[37] M. Eismann, R. Hardie, Application of the stochastic mixing model to hyperspectral resolution enhancement, IEEE Transac- tions on Geoscience and Remote Sensing 42 (9) (2004) 1924-1933.

[38] C. Pohl, J. Van, Genderen, "Multisensor image fusion in remote sensing: concepts methods and applications," International Journal of Remote Sensing, Vol. 19(5), pp 823-854, 1998.

[39] R. A. Schowengerdt,Remote Sensing: Models and Methods for Image Processing, 2nd ed. Orlando, FL, Academic, 1997.

[40] Z. Wang, D. Ziou, C. Armenakis, Q. Li, ”A Comparative Analysis of Image Fusion Methods," IEEE Trans. Geoscience remote sensing, vol. 43, no. 6, pp. 1391-1402, 2005.

[41] Tania, Stathaki, Image Fusion: Algorithms and Applications, Elsevier, 1st ed., 2008.

[42] PouranBehnia, "Comparison Between Four Methods for Data Fusion of ETM+ Multispectral and Pan Images," Journal of Geo-spatial Information, Vol. 8,Issue 2, 2005.

[43] MultiSpec@,https://engineering.purdue.edu/ biehl/MultiSpec/hyperspectral.html 